\title{
Effects of stimulus concreteness-imagery and arousal on immediate and delayed recall*
}

\author{
JOHN C. SCHMITT and WILLIAM E. FORRESTER \\ State University College at Oswego, N.Y. 13126
}

Data reported by Butter (1970, Experiment II) indicate that effects of stimulus concreteness-imagery on incidental recall may reverse over a long retention interval. Consistent with previous results, Butter also found a reversal of stimulus-arousal effects over time. In the present attempt to replicate Butter's findings, no reversal was found for either the concreteness-imagery or arousal variable. A strong concreteness-imagery main effect was found, however, with high stimulus concreteness-imagery facilitating recall on both immediate and delayed tests.

A number of studies, beginning with that of Kleinsmith \& Kaplan (1963), have shown that incidental recall of paired associates is affected by arousal value of the stimulus terms. Responses paired with low-arousal stimuli are recalled better than those paired with high-arousal stimuli on an immediate retention test, but the effect reverses after a longer retention interval. Results of a study by Butter (1970, Experiment II) suggest that a similar reversal occurs with respect to the effects of stimulus concreteness (C) and imagery value (I).

The specific procedures used by Butter were to present a list of eight paired associates to Ss, with half of the pairs having high and half having low C-I stimuli. For Ss tested 2 min after list presentation, recall of responses paired with high C-I stimuli was better than recall of responses paired with low C-I stimuli. For Ss tested 2 days after list presentation, however, the effect was reversed. Since Butter had obtained GSR data from her Ss during stimulus presentation, she was also able to determine the effects of arousal on retention. Analysis of the retention data, with respect to arousal effects, showed the pattern of recall to be in agreement with that of Kleinsmith \& Kaplan (1963) and just the reverse of that associated with the C-I variable. Consistent with the retention results, Butter reported additional data indicating that low C-I items tended to be classified as high arousal, while high C-I items were low in arousal.

Yuille (1971) attempted to replicate Butter's findings with respect to the reversal of C-I effects, but was unable to do so. Yuille suggested two possible reasons for this failure to replicate. One possibility is that Butter's monitoring of GSRs, a procedure not followed by Yuille, may have modified the learning situation in some way. The second possibility suggested by Yuille is that Butter had made an error in her analysis of C-I effects. On the basis of recall percentages reported by Butter,

\footnotetext{
*This research was supported in part by Grant HD06420 from the National Institute of Child Health and Human Development to the second author.
}

Yuille concluded that only 12 Ss per group were included in the analysis rather than the 16 Ss per group Butter had reported testing. While Yuille's observation that Butter had omitted data from analysis appears to be correct, the suggestion that Ss had been eliminated is contraindicated by the values Butter reports as degrees of freedom in her analysis of variance. A more likely possibility is that two list items were eliminated from each S's retention data. In her analysis of arousal effects, Butter did specify that the stimulus items were rank ordered for each $\mathbf{S}$ on the basis of GSR deflections, and the items occupying Ranks 4 and 5 were eliminated from analysis. While a similar procedure could have been used with respect to C-I effects, it is not clear whether items were eliminated because they ranked 4 and 5 on the concreteness dimension or on the imagery dimension. Rank ordering of Butter's eight stimuli on the basis of Paivio, Yuille, \& Madigan's (1968) norms shows that different items occupy Ranks 4 and 5 on the two dimensions.

The purpose of the present study was to provide a further investigation of the effects reported by Butter (1970). The primary difference between the -present study and Yuille's (1971) attempted replication of Butter is that measurement of GSRs was incorporated into the present procedures. With regard to analysis of C-I effects, however, the procedures followed in the present study were consistent with Yuille in that no data were excluded.

\section{METHOD}

An eight-item paired-associate list was used as the learning material. Stimulus terms, taken from Butter (1970), were the words GIRL, HARP, NAIL, LIBRARY, ATTITUDE, SOLL, DEMOCRACY, and THEORY. The first four items comprised the high C-I sublist with mean $C$ and I values of 6.89 and 6.68 , respectively, according to Paivio, Yuille, \& Madigan's (1968) norms. The last four items, the low $\mathrm{C}$-I sublist, have mean $\mathrm{C}$ and I values of 1.85 and 2.49 , respectively. Response terms were the single digits 2-9. Two randomly determined sets of word-digit pairings were used in the study. In addition to the learning materials, multicolored slides (four colors per slide) were constructed for use as filler items.

The Ss were 32 undergraduates enrolled in introductory psychology classes at Oswego State. None had served in any previous verbal learning or memory experiments. Assignment of Ss to the two sets of word-digit pairings and to retention-interval conditions ( 2 min or 2 days) was determined by a randomized schedule and by the Ss' order of appearance at the laboratory.

Prior to the training sequence. Ss were instructed to concentrate on all slides and to call out the colors, words. and digits as they were presented. For the training sequence. each stimulus word was first presented alone and then presented with its corresponding response digit. Two filler slides appeared in between presentation of a word-digit pair and the succeeding stimulus word. Before the recall sequence began. Ss were again instructed to call out the colors on the filler slides and. when a word was presented. to say the digit that had previously been 
Table 1

Percentage and Absolute Number (in Parentheses) of Paired Associates Recalled

\begin{tabular}{lllrl}
\hline & \multicolumn{4}{c}{ Retention Interval } \\
\cline { 2 - 5 } Stimulus & \multicolumn{2}{c}{2 Min } & \multicolumn{2}{c}{2 Days } \\
\hline High C-I & 28.13 & $(18)$ & 21.88 & $(14)$ \\
Low C-I & 18.75 & $(12)$ & 4.69 & $(3)$ \\
High Arousal & 25.00 & $(12)$ & 12.50 & $(6)$ \\
Low Arousal & 20.83 & $(10)$ & 12.50 & $(6)$ \\
\hline
\end{tabular}

paired with that word. For the recall sequence, three filler slides were presented between successive stimulus words with the word-digit slides being omitted.

All items were presented by a Carousel projector with an automatic timer set to $4 \mathrm{sec}$ between onset of consecutive slides. Two randomized orders of items were used for the training sequence, and two different randomizations were used for recall. During training and recall, GSR data were recorded by means of a physiograph, operating at a paper speed of $.25 \mathrm{~cm} / \mathrm{sec}$.

\section{RESULTS AND DISCUSSION}

Recall of responses paired with high and low C-I stimuli is shown in Table 1. Preliminary analyses indicated that assumptions underlying use of analysis of variance could best be met by subjecting the present data to a Freeman-Tukey transformation. Analysis of variance performed on the transformed data indicated a significant main effect for C-I, $F(1,30)=9.58, p<.005$. The retention interval main effect and C.I by Retention Interval interaction approached significance, $p<.07$ and $\mathrm{p}<.06$, respectively.

The results concerning C-I thus indicate a strong effect persisting over the 2-day retention interval. The reversal of C-I effects reported by Butter (1970) was not supported by the present data. In this respect, the results of the present study are in agreement with those of Yuille (1971). Although Yuille (1971) did not find significant C-I effects following the 2-day retention interval, he did report data from two previously unpublished experiments in which the effects of stimulus concreteness (in an intentional-learning task) persisted over an interval of 1 week.

In order to determine effects of the arousal variable in the present study, the data were first quantified in a manner consistent with previous research on this variable. Specifically, the eight stimulus items were rank ordered for each $\mathrm{S}$ on the basis of GSR deflections occurring within a $12 \cdot \mathrm{sec}$ period following presentation of each stimulus. The three items that produced the greatest deflections were classified as high arousal, and the three that produced the smallest deflections were classified as low arousal.

As may be seen in the bottom half of Table 1, there was virtually no difference between recall of high- and low-arousal pairs at either retention interval. Analysis of variance indicated that neither the main effect of arousal nor the Arousal by Retention Interval interaction was significant. The main effect of retention interval approached significance, $F(1,30)=3.33, p<.10$.

The failure to find a reversal of arousal effects over a 2-day retention interval is inconsistent with previous research in the area. One reason for this failure may be that the items used in the present study do not form clear-cut high- and low-arousal classes. A second possibility is that the effects of stimulus concreteness and imagery associated with the present items obscured the potential effects of the arousal variable. It should be noted that Butter (1970) did find a reversal of arousal effects with these items. Yuille (1971) did not analyze for arousal effects. If, however, Butter's conclusion that arousal and C-I are inversely related is correct, then it must follow that Yuille's data do not reflect a reversal of arousal effects either.

\section{REFERENCES}

Butter, M. J. Differential recall of paired associates as a function of arousal and concreteness-imagery levels. Journal of Experimental Psychology, 1970, 84, 252-256.

Kleinsmith, L. J., \& Kaplan, S. Paired-associate learning as a function of arousal and interpolated interval. Journal of Experimental Psychology, 1963, 65, 190-193.

Paivio, A., Yuille, J. C., \& Madigan, S. Concreteness, imagery, and meaningfulness values for 925 nouns. Journal of Experimental Psychology, 1968, 76 (1, Part 2).

$Y$ uille, J. C. Does the concreteness effect reverse with delay? Journal of Experimental Psychology, 1971, 88, 147-148.

(Received for publication April 9, 1973.) 